\title{
Dynamical System Algorithm Specification Analysis and Stabilization
}

\author{
Charles C. Phiri ${ }^{1,2}$, János Botzheim ${ }^{2,3}$, Cristina Valle ${ }^{2}$, Zhaojie $\mathrm{Ju}^{1,4 *}$, and \\ Honghai $\mathrm{Liu}^{1}$ \\ 1 School of Computing, University of Portsmouth, UK, \\ zhaojie.ju@port.ac.uk \\ 2 CC Initiative Ltd, UK \& Japan, \\ 3 Department of Automation, Széchenyi University, Győr, Hungary, \\ ${ }^{4}$ School of Automation, Wuhan University of Technology, China,
}

\begin{abstract}
This paper investigates approaches to deliberately designing systems whose controllability can be quantified. Preliminary findings of ongoing research are presented on complex dynamical system control algorithms. The specification analysis and quality of the pressure control algorithm applied to a Topical Negative Pressure Wound Therapy device are conducted, with further discussion on self-regulation mechanism and characterization of both the partially observable and partially controllable workspace represented by the negative pressure chamber. Statistical methods are employed to understand the device physics and fuzzy logic and bacterial memetic algorithm are utilised to explore and optimize the existing algorithms and also extract the rule base.
\end{abstract}

Keywords: Specification Analysis, Fuzzy Inference, Bacterial Memetic Algorithm

\section{Introduction}

In highly regulated environments, such as the medical device industry, designing complex dynamical systems is usually prescribed as an exact art. This, in reality, is not the case and many medium sized companies settle for the minimum acceptable solution in order to minimize Research and Development costs or the cost of hiring domain expert help. On the other hand, the complexity of the system may not be fully understood by the development team such that decisions based on a dogmatic view of the world usually pass as the pragmatic solution. In this paper, we examine approaches to deliberately designing systems whose controllability can be quantified. We present the preliminary findings of ongoing research on complex dynamical system control algorithms. We further discuss the specification analysis and quality of the pressure control algorithm applied to a Topical Negative Pressure Wound Therapy device, NPWT [1]; we also look at self-regulation mechanism and characterization of both the partially observable and partially controllable workspaces represented by the negative pressure chamber. 
Dynamical systems theory and chaos theory deal with the long-term qualitative behavior of dynamical systems [6]. The task in this paper is not to try and solve the intractable problem of finding a precise solution to the set of equations describing the dynamical system, but rather we are seeking to provide answers to the questions of whether the system settles down to a steady state in the long term. In the event that the system does indeed settle down, what are the possible steady states and whether the long-term behavior of the system depend on its initial condition. We use statistical methods to understand the device physics; we apply fuzzy logic [11] and Bacterial Memetic Algorithm (BMA) [3, 4] to explore and optimize the existing pressure control algorithms. BMA is used to infer the fuzzy rule base to identify the optimal control parameters and strategy.

\section{Topical Negative Pressure Wound Therapy Pump as a Dynamical Systems}

The concept of a dynamical system has its origins in Newtonian mechanics [6]. The evolution rule of dynamical systems is an implicit relation that gives the state of the system for only a short time into the future. The rule describes the time dependence of the position of a point in its ambient space represented as a vector mapped to the coordinates of a manifold (geometrical space). To determine the state for all future times requires iterating the relation many times for each advancing small time step. The subsequent step from the current step are described by a fixed rule known as the evolution rule. The rule may be deterministic such that for a given time interval only one future state follows from the current state; or it may be stochastic such that the evolution of the state is subject to random shocks. Solving the dynamical system (or integrating the system) to determine the states at all future time frames involves iterating the map many times for each advancing small time step. If the system can be solved, given an initial point, it is possible to determine all its future positions, a collection of points known as a trajectory or orbit. Sharkovsky's theorem [2, $10]$ is an interesting statement about the number of periodic points of a onedimensional discrete dynamical system. A dynamical system can be viewed as a study of iterations of a continuous function from an interval into itself.

Figure 1 shows the lumped parameters model of the system. This is a logical model of the system capturing both the controllable and observable components of the pneumatic circuit. The pressure present at the wound site ideally represents the delta between the pressure measurements in pressure sensor alpha and pressure sensor beta. In the control loop, these can either be used as individual input parameters or combined by observing the delta; this describes a completely different characteristic to the individual inputs. Due to the positioning of the pressure sensors, the presence of pressure release valves, the fact that the wound contact dressing does not form a perfect seal, the effects of gravity on fluids in the lumen and changes in its diameter; the varying ratios of exudate to air, viscosity of the fluids and their biochemical composition and reactions; the system under observation is not fully observable and exhibits turbulent flow. 
The observed pressure includes noise introduced from both intrinsic variances and nonlinearities in the sensors and ADC's in the loop which contribute directly to the accuracy of the measurement method and the statistical characteristics of the observable ambient space. The top left of the figure shows the model of the effects fluid flow in a lumen.

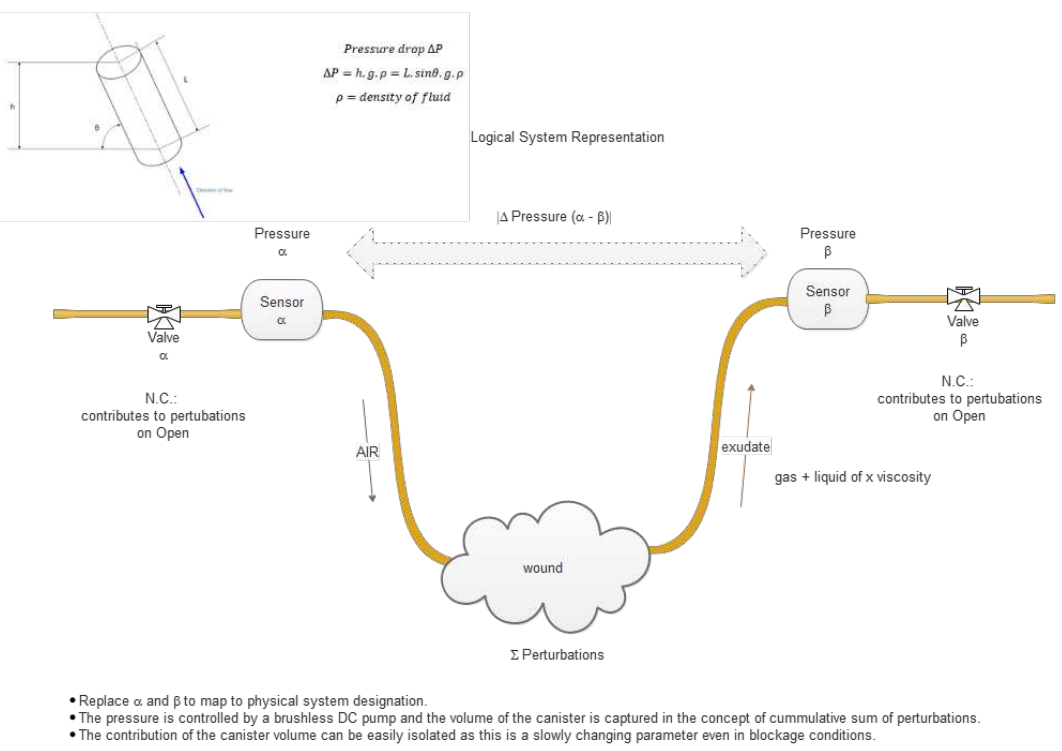

Fig. 1. Lumped Parameters Model

In NPWT a double lumen (or multi lumen) system has the possibility to (1) introduce a defined air leak into the system to create the pressure delta required to move fluid and (2) independently monitor the pump pressure and wound pressure to allow the system to determine to adjust the pump pressure accordingly. The air leak is not always necessary, on the contrary a large constant air leak increases the load on the vacuum pump resulting in more noise and drawing more power from the battery. Monitoring both the pump pressure and wound pressure helps determine when the introduction of an air leak would be beneficial. The balancing exercise is to let air in when it is needed, meaning when the risk exists for exudate standing still in the tubing. To accommodate this proposed self-regulation, the system is equipped with 2 pressure sensors and 2 valves in the pneumatic circuit.

Figure 2 shows the system decomposed further to a conceptual model that looks at the mapping between the physical world to control theory and dynamic programming. This allows the solutions to be developed without too much noise 
from the details. The two worlds are coupled and adjusted in tandem to make sure that the model evolves in step with the availability of expert knowledge of the physical system. This also provides a mapping of the specific terms and environments owing to the pedigree of the theories themselves. The system is modeled using function approximators that treat the concept as a discrete system. This demands that a mathematical model of the system exists beforehand. While dynamic programing model requires that a model is present in the first place, reinforcement learning model does not require that the model exist beforehand. The features of the system are learnt during the evolution cycles of the system. The state space is summarized in the bottom left of the figure; this shows how the transitions occur during the iterations. The bottom right captures the essence of the entire problem space in terms of an artificial intelligent agent. In this view the system is collapsed into a collection of sensors perceiving the configuration space and a set of control actions influencing the configuration space. The models are all contingent on the existence of the feedback loop.

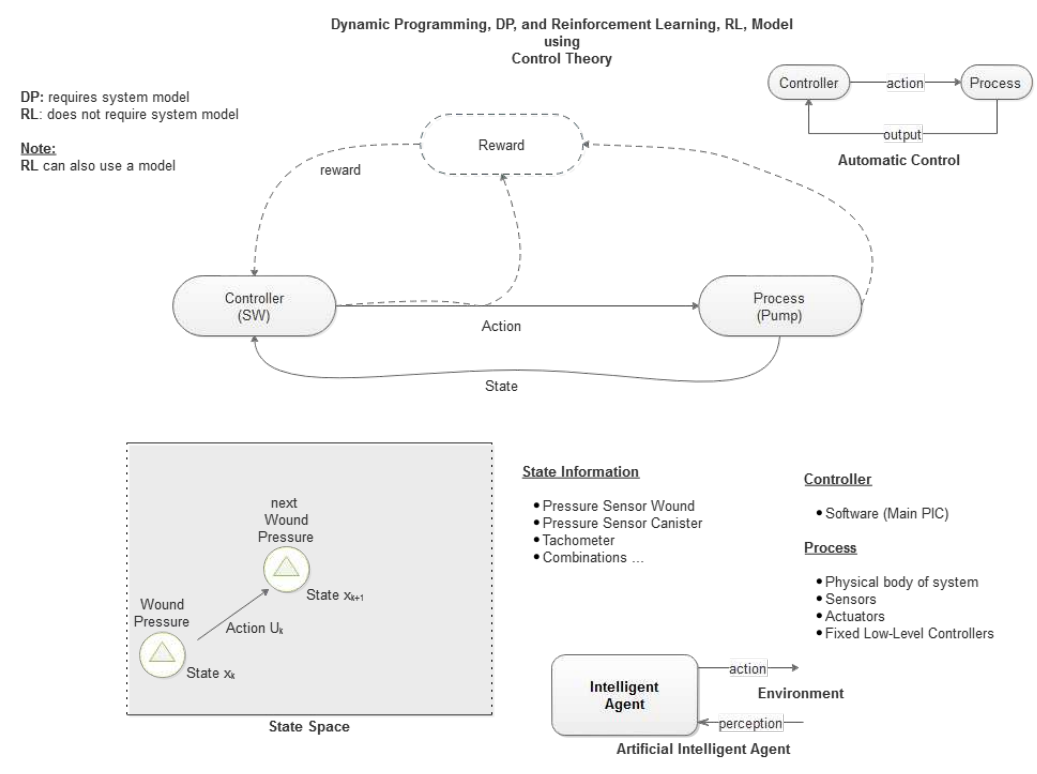

Fig. 2. State Space Model

The concept starts by exploring whether a method exists to consistently and efficiently determine if a model fits the specification and if so, by what metric to make that determination. In order to appreciate the extent of the challenge, we start by restating the problem space and bounding it to the following terms: 
- Obtain a quantifiable description of the pump and the control algorithm

- Identifying dominant features in the pump performance and characteristics

- Describe the relationships between the features (duty cycle of the PWM control signal for the pump, pump speed, wound and canister pressure readings)

- Describe characteristic performance at the steady state

- Describe characteristic performance in the transient state

The NPWT system describes a leaky system, the control trajectory includes an error term. The flow of fluids in the pneumatic circuit of the NPWT may exhibit chaotic behavior where small initial errors in a trajectory may diverge exponentially. The control algorithm focuses on moving the system towards a stable operating point. On the other hand, the effects of the control mechanisms are not immediately applied (temporal term). The system is unstable without control. The dynamics of the system are unstable. The effect of the control commands have a temporal delay. The effects are observable.

We state here without proof that the NPWT is a nonlinear dynamical system. The regulation algorithm for the NPWT tries to achieve the asymptotic stability of the nonlinear systems. Plenty of literature exists describing the control theory in terms of the Lyapunov Stability [8]. The range of control mechanisms in the industry range from Bang-Bang control through evolved versions of PID to discrete finite state control system with varying levels of performance. In BangBang control (hysteresis control), an envelope is set around the set-point and the control signal is applied in such a way that the response signal oscillates between the upper and lower bounds of the envelope. The thresholds are set as of crisp values which when reached trigger an alteration in the state of the control signal. Lyapunov's stability analysis is applied to design globally asymptotically stable, nonlinear feedback controllers. The control law saturation is described as follows:

$$
u(t)= \begin{cases}U_{\max } & \text { if } u_{\text {des }}(t) \geq U_{\max } \\ u_{\text {des }} & \text { if } U_{\min }<u_{\text {des }}(t)<U_{\max } \\ U_{\min } & \text { if } u_{\text {des }}(t) \leq U_{\min }\end{cases}
$$

The outcome of this research is not sensitive to a specific control mechanism. The control algorithms in this paper included classical PID and FSM. The methodology however is not specific to these algorithms, the selection was determined by existing implementations. The advantage of using these algorithms is they can easily be described using accessible mathematical models, which in turn allowed easily accessible validation of the effectiveness of the control mechanism.

The working hypotheses are stated as below:

- Given a set point (the nominal therapy condition at a suction of $-120 \mathrm{mmHg}$ delivered at the wound site):

- it is possible derive the function mapping the input parameters to the output parameters

- it is possible to map out an optimal control strategy

- The control signal can be made adaptive based on the deviation of the output compared to the steady-state error at the wound site 
6 Charles C. Phiri et al.

- The regulation algorithm can be modified to maintain the range of the delta of the pressure sensor outputs within bounded limits

\section{Specification Analysis using Fuzzy Rule Base}

The performance criteria of the NPWT and the majority of medical devices is strictly regulated by the relevant authorities especially in situations where there is a risk of injury or death to the patient. The control software development has to comply with the IEC/ISO/IEEE 62304 Medical Device Software Life Cycle process, at the very least. The risk is mitigated via the application of clinical domain expert knowledge as well as the resolution of the the ISO 14971 Medical Devices Application of Risk Management to Medical Devices. Institutions such as the Food and Drug Authority, FDA, provide guidance and enforcement for products entering the US markets. These standards and risk control measures and the subsequent test methods and methodologies care about whether the system works for the specified range of safe parameters or if it is broken. In this research case, however, focus on metric with which to measure the if the model fits to the specification. Combining the critical components in the standards and system specification, in our research, the linguistic variables (computable information granules) could be extracted and modeled. These variables were then manually translated into a Fuzzy Rule Base. Figure 3 shows the surface graph. From this specification it was established that pump was over-specified by a factor of approximately $60 \%$. This directly contributed to tighter control measures being applied to remove the risk of a runaway system that could result in extreme injuries to the patient. Hardware controls feature have been implemented to minimize this risk.

Also from Figure 3, it can be shown that the system performance will always fall within the surface graph. The boundary of discrimination is defined by the aforementioned linguistic variables and coincide with the boundary for safely operating the pump in this configuration to deliver adequate therapy. Further, as there exists a direct relationship between the control voltage applied to the pump and the duty cycle; the pump being one of the largest contributors to the power budget of the unit, the description of the duty cycle in this figure closely approximates the power performance response graph. Further, this also defines the surface area over which the system could be safely customized.

\subsection{Checking Assumptions}

Given that no mathematical model exists, the control system has to be validated by using experimental data. Figure 4 shows the relationship between duty cycle and pump speed (RPM). It is therefore not surprising that there is a very strong correlation between the pump duty cycle and the pump speed. Figure 5 looks at the relationship between the pressure sensors alpha and beta. The lumina (lumens), represented by the two pressure sensor reading are controlled by a suction generated from a single pump. It should be noted that owing to the physics of 


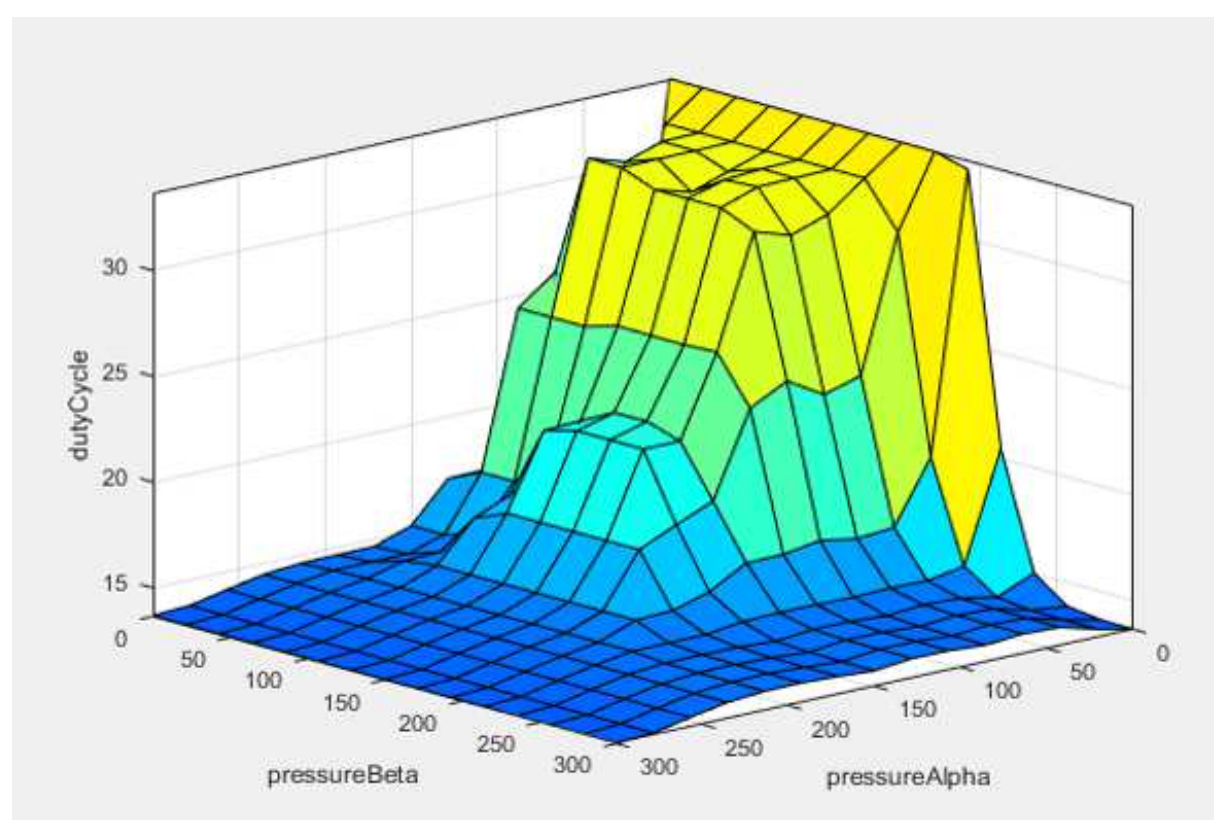

Fig. 3. Specification Analysis

the device, the lumina are out of phase. Pressure Sensor Alpha response has the effect of applying a low pass filter on the Pressure Sensor Beta response graph. In other words, the perturbations in beta lumen are attenuated (and inverted) in alpha lumen response graph.

\subsection{Computational Intelligence Based Model}

Based on above assumptions, a computational intelligence based model is proposed as illustrated in Fig. 6. In this model, a fuzzy rule base is extracted and optimized. The inputs of the fuzzy rule base are the pressure sensors alpha and beta. The pump RPM value can be omitted from the inputs because its relationship to the duty cycle as shown in Fig. 4. The output of the fuzzy rule base is the percentage duty cycle. Mamdani type fuzzy rules and inference method are applied in the fuzzy model [5].

The optimization of the fuzzy rule base is performed by Bacterial Memetic Algorithm which is a population based stochastic nature-inspired optimization algorithm [3,4]. BMA effectively combines global and local search in order to find a quasi-optimal solution for the given problem. The bacterial operators (bacterial mutation and gene transfer) are applied in the global search. The role of the bacterial mutation is the optimization of the bacteria's chromosome. The gene transfer allows the transfer of information in the population among the different bacteria. As a local search technique, the Levenberg-Marquardt method is applied by a certain probability for each individual. One rule base corresponds 


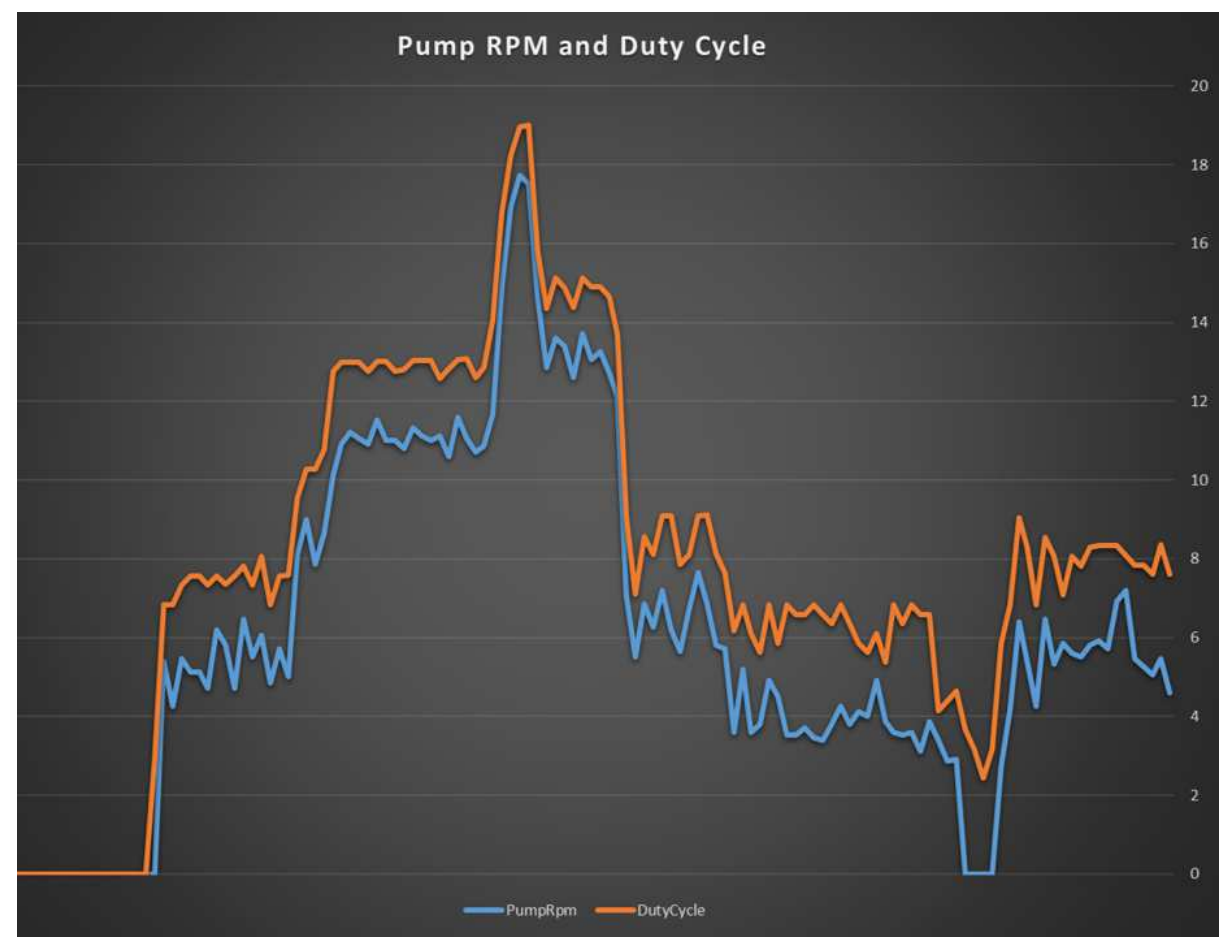

Fig. 4. Pump Rpm and Duty Cycle 


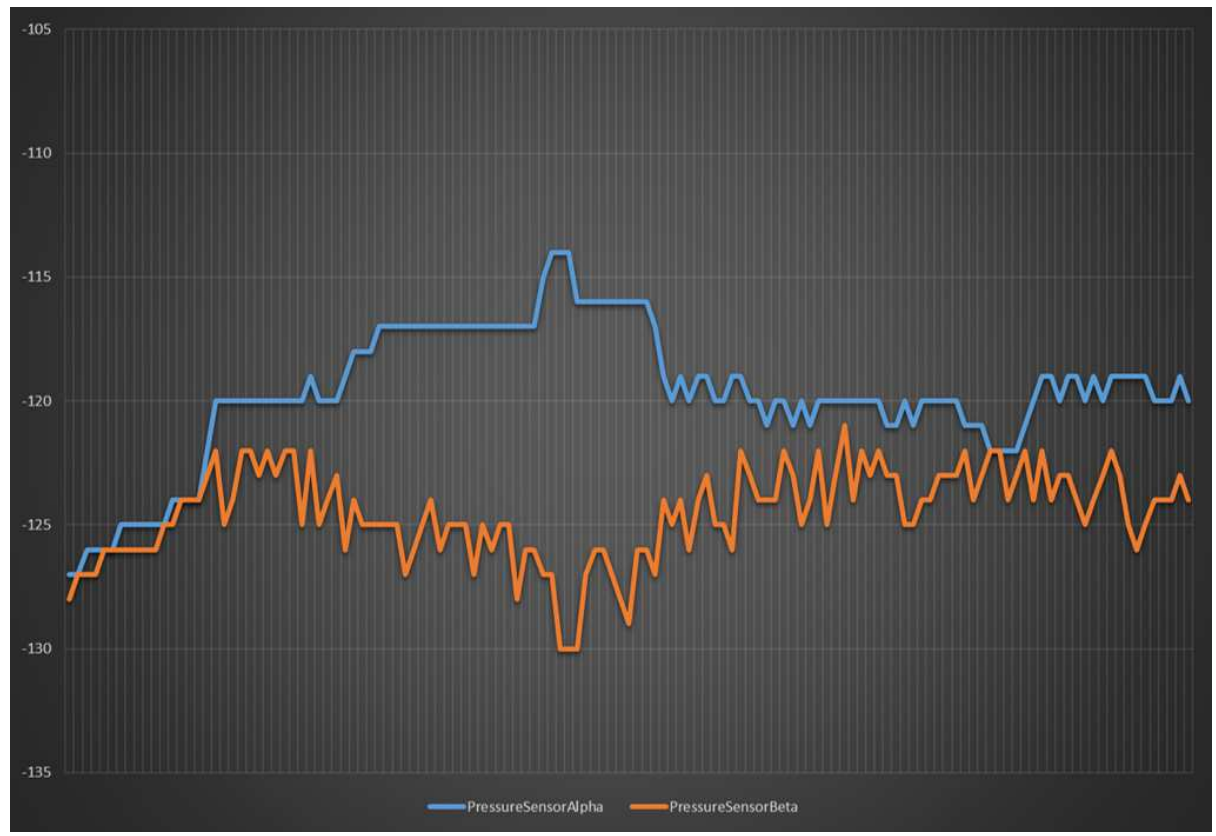

Fig. 5. Pressure Alpha and Pressure Beta Relationship

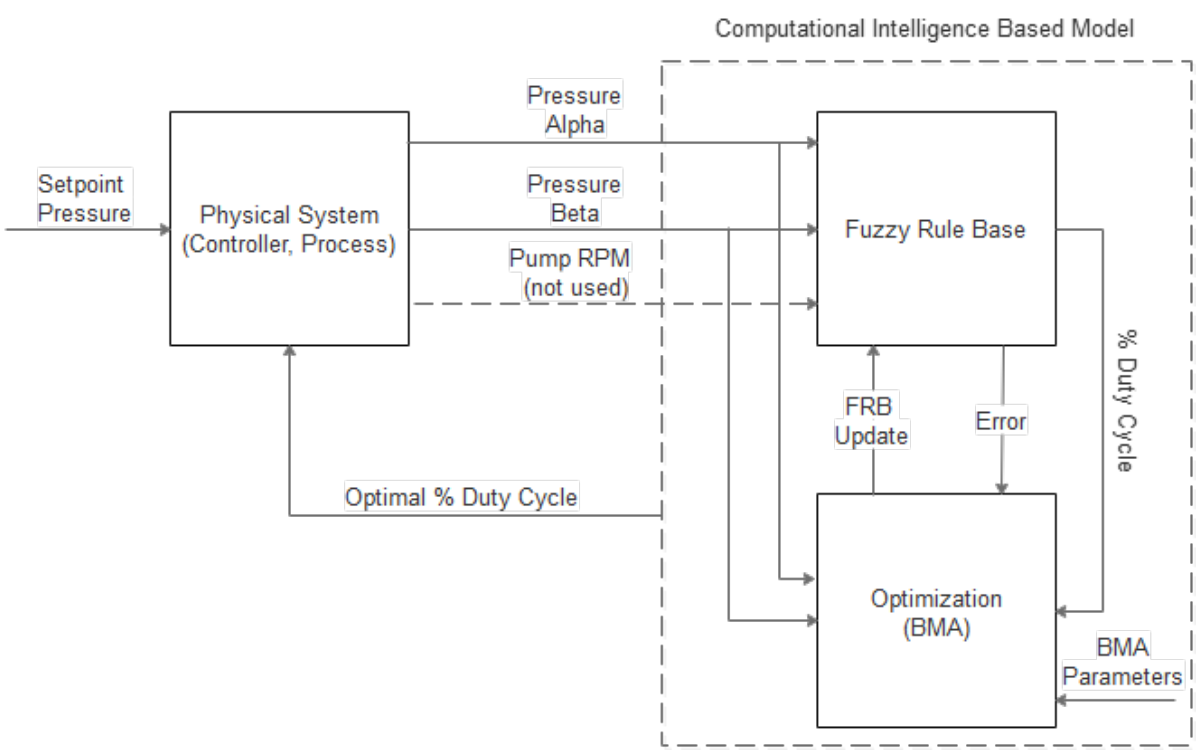

Fig. 6. Computational Intelligence Based Model 
to one bacterium in the BMA when applied for fuzzy rule base optimization. The fuzzy rule bases (bacteria) are evaluated by the mean square error criterion. BMA updates the fuzzy rule base parameters (membership functions) by the bacterial mutation, Levenberg-Marquardt, and gene transfer operations [3].

The output of the computational intelligence based model is the optimal percentage duty cycle which is fed back into the physical system.

\section{Conclusion}

In this work, we have shown approaches to deliberately designing systems which are partially controllable and partially observable. We have presented the preliminary findings of ongoing research on complex dynamical system control algorithms. This research uses data from the medical industry. We have discussed the specification analysis and quality of the control algorithm applied to a Topical Negative Pressure Wound Therapy device, and we have also looked at selfregulation mechanism and characterization of both the partially observable and partially controllable workspace represented by the negative pressure chamber. We have proposed a computational intelligence based model to determine the optimal percentage duty cycle value for the physical system. In the computational intelligence based model a fuzzy rule base approach has been used together with a nature-inspired optimization algorithm. Although the proposed approach was not able to fully solve the optimal control of the pump, it encourages us for further research. As a future work our aim is to deeply investigate the relationship between the duty cycle and the pressure of the pump by computational intelligence based models $[7,9]$.

Acknowledgments. The authors would like to acknowledge support from CC Initiative Ltd, project of NSFC (51575412), DREAM EU FP7-ICT (611391), State Key Laboratory of Digital Manufacturing Equipment \& Technonlogy (DMETKF2017003), and the exchange program from School of System Design, Kubota Laboratories, Japan.

\section{References}

1. Berrevoet, F., Vanlander, A., Sainz-Barriga, M., Rogiers, X., Troisi, R.: Infected large pore meshes may be salvaged by topical negative pressure therapy. Hernia 17(1), 67-73 (2013)

2. Borwein, J., Bailey, D.: Mathematics by Experiment: Plausible Reasoning in the 21st Century. A K Peters, Wellesley, Massachusetts (2003)

3. Botzheim, J., Cabrita, C., Kóczy, L.T., Ruano, A.E.: Fuzzy rule extraction by bacterial memetic algorithms. International Journal of Intelligent Systems 24(3), 312-339 (March 2009)

4. Botzheim, J., Toda, Y., Kubota, N.: Bacterial memetic algorithm for offline path planning of mobile robots. Memetic Computing 4(1), 73-86 (2012) 
5. E.H. Mamdani, S.A.: An experiment in linguistic synthesis with a fuzzy logic controller. International Journal of Man-Machine Studies 7(1), 1-13 (1975)

6. Gotoda, H., Shinoda, Y., Kobayashi, M., Okuno, Y., Tachibana, S.: Detection and control of combustion instability based on the concept of dynamical system theory. Physical Review E 89(2), 022910 (2014)

7. Ju, Z., Liu, H., Xiong, Y.: Fuzzy empirical copula for estimating data dependence structure. International Journal of Fuzzy Systems 16(2), 160-172 (2014)

8. Jung, J.W., Leu, V.Q., Do, T.D., Kim, E.K., Choi, H.H.: Adaptive pid speed control design for permanent magnet synchronous motor drives. IEEE Transactions on Power Electronics 30(2), 900-908 (2015)

9. Phiri, C.C., Ju, Z., Liu, H.: Accelerating humanoid robot learning from human action skills using context-aware middleware. In: International Conference on Intelligent Robotics and Applications. pp. 563-574. Springer (2016)

10. Weisstein, E.: Sharkovsky's theorem. Mathworld-a Wolfram web resource. http: //mathworld.wolfram.com/SharkovskysTheorem.html

11. Zadeh, L.: Fuzzy sets. Information and Control 8, 338-353 (1965) 